\title{
TRAVELING WAVES FOR A NONLOCAL DISPERSAL SIR MODEL WITH STANDARD INCIDENCE
}

\author{
WAN-TONG LI AND FEI-YING YANG \\ Communicated by Stig-Olof Londen
}

\begin{abstract}
This paper is concerned with traveling wave solutions of a nonlocal dispersal SIR epidemic model with standard incidence. We show that our results on existence and nonexistence of traveling wave solutions are determined by the basic reproduction number of the corresponding ordinary differential model and the minimal wave speed. These threshold dynamics are proved by constructing an invariant cone and applying Schauder's fixed point theorem on this cone and the Laplace transform. The main difficulties are the lack of an occurrence of a regularizing effect and the loss of the order-preserving property of this model.
\end{abstract}

1. Introduction. The current paper is concerned with the existence and nonexistence of traveling wave solutions of the following nonlocal dispersal SIR model with standard incidence:

$$
\left\{\begin{array}{l}
\frac{\partial}{\partial t} S(x, t)=d_{1}(J * S(x, t)-S(x, t))-\frac{\beta S(x, t) I(x, t)}{S(x, t)+I(x, t)}, \\
\frac{\partial}{\partial t} I(x, t)=d_{2}(J * I(x, t)-I(x, t))+\frac{\beta S(x, t) I(x, t)}{S(x, t)+I(x, t)}-\gamma I(x, t), \\
\frac{\partial}{\partial t} R(x, t)=d_{3}(J * R(x, t)-R(x, t))+\gamma I(x, t),
\end{array}\right.
$$

where $S, I$ and $R$ denote the sizes of the susceptible, infected and removal individuals, respectively. The infection rate $\beta$ and the removal rate $\gamma$ are positive numbers. $d_{i}>0(i=1,2,3)$ are dispersal rates for the susceptible, infected and removal individuals, respectively. $J * S(x, t), J * I(x, t)$ and $J * R(x, t)$ are the standard convolutions with

2010 AMS Mathematics subject classification. Primary 35K57, 35R20, 92D25.

Keywords and phrases. Traveling waves, nonlocal dispersal, SİR model, Schauder's fixed point theorem, Laplace transform.

Research supported by NSF of China (Nos. 11031003 and 11271172) and FRFCU (No. lzujbky-2011-k27).

The second author is the corresponding author. 2013.

Received by the editors on December 15, 2012, and in revised form on May 16, 
space invariable $x$. Throughout this paper, we assume that the nonlocal dispersal convolution kernel $J$ is a smooth function on $\mathbf{R}$ and satisfies:

$$
\begin{gathered}
J \in C^{1}(\mathbf{R}), \quad J(x)=J(-x) \geq 0 \\
\int_{\mathbf{R}} J d y=1 \quad \text { and } \quad J \text { is compactly supported. }
\end{gathered}
$$

It is well known that disease propagation in space is relevant to the socalled traveling waves, solutions of the form $(S(x+c t), I(x+c t), R(x+$ $c t)$ ) for which $c$ is called the wave speed. For applications to disease control and prevention, it is important to determine whether traveling waves exist and what the propagation speed $c$ is. Moreover, it is found that the first and second equations of system (1.1) form a closed system, it suffices to consider a two-dimensional system for $S$ and $I$.

System (1.1) is the nonlocal counterpart of the following SIR disease outbreak model with the standard incidence

$$
\left\{\begin{array}{l}
\frac{\partial}{\partial t} S=d_{1} \frac{\partial^{2} S}{\partial x^{2}}-\frac{\beta S I}{S+I}, \\
\frac{\partial}{\partial t} I=d_{2} \frac{\partial^{2} I}{\partial x^{2}}+\frac{\beta S I}{S+I}-\gamma I, \\
\frac{\partial}{\partial t} R=d_{3} \frac{\partial^{2} R}{\partial x^{2}}+\gamma I .
\end{array}\right.
$$

This model was considered by Wang et al. [35]. They showed that if $R_{0}:=\beta / \gamma>1$, then for each $c>c_{0}=2 \sqrt{d_{2}(\beta-\gamma)}$, the system

$$
\left\{\begin{array}{l}
\frac{\partial}{\partial t} S=d_{1} \frac{\partial^{2} S}{\partial x^{2}}-\frac{\beta S I}{S+I}, \\
\frac{\partial}{\partial t} I=d_{2} \frac{\partial^{2} I}{\partial x^{2}}+\frac{\beta S I}{S+I}-\gamma I
\end{array}\right.
$$

has a traveling wave solution $(S(x+c t), I(x+c t))$ and no traveling wave solutions for $0<c<c_{0}$. On the other hand, there are no traveling wave solutions for $R_{0}=\beta / \gamma \leq 1$. The proof is mainly based on that of Wang and $\mathrm{Wu}[\mathbf{3 8}]$ and several earlier studies, see $[\mathbf{1 3}, \mathbf{2 0}, \mathbf{2 3}, \mathbf{3 6}$, 37]. Here, $R_{0}=\beta / \gamma$, which is the so-called basic reproduction number calculated from the corresponding ordinary differential system of (1.3) at the initial disease-free equilibrium $\left(S_{0}, 0,0\right)$, completely determines the transmission dynamics and epidemic potential: if $R_{0}>1, I(t)$ increases to its maximum firstly and then decreases to zero and hence an epidemic takes place; if $R_{0}<1$, then $I(t)$ decreases to zero and the epidemic does not occur. 
Systems (1.1) and (1.3) describe the transmission of communicable disease. Classically, the random (or local) dispersal operator is common in modeling the diffusion of the species; it suggests that the population at location $x$ can only be influenced by the variation of the population near the same location $x$, see $[\mathbf{1}, \mathbf{1 1 - 1 5}, \mathbf{1 9}, \mathbf{2 4}, \mathbf{2 9}, \mathbf{3 8}, \mathbf{4 0}, 42]$. However, the random (or local) dispersal operator such as the Laplace operator can only influence a species' immediate neighborhood for ecological and epidemiological models. One method in overcoming these problems with the Laplace operator is to describe these models connecting spatial migration by a nonlocal dispersal operator, see $[15,17,18,23]$. For traveling wave solutions of nonlocal dispersal problems, we refer to $[\mathbf{3}-\mathbf{8}, \mathbf{1 0}, \mathbf{2 1}, \mathbf{2 5 - 2 8 ,} \mathbf{3 0 - 3 2 , 3 4 , 4 3 - 4 9 ]}$ and references therein. In addition, one refers to $[\mathbf{2}, \mathbf{9}, \mathbf{1 6 - 1 8 , 3 3}]$ to understand the development of this type of work.

In the present paper, we focus on the existence and nonexistence of traveling wave solutions of system (1.1). Note that system (1.1) does not satisfy the mixed quasimonotone or exponential mixed quasimonotone conditions and the method in $[\mathbf{2 2}, \mathbf{2 7}, \mathbf{2 8}]$ may not be used directly to consider the existence of traveling wave solutions any longer. It is known that Schauder's fixed theorem has been used to prove the existence of nontrivial traveling wave solutions by constructing a suitable invariant set in the past years, see $[\mathbf{1 3}, \mathbf{2 0}, \mathbf{2 3}, \mathbf{3 8}]$. Inspired by $[\mathbf{1 2}$, 13, 39], we want to prove the existence of nontrivial traveling wave solutions by constructing an invariant cone in a large bounded domain with the initial functions being defined on and applying Schauder's fixed theorem on this cone, then passing to the unbounded domain by a limiting argument. But, we find that a uniform a priori bound of solutions of nonlocal dispersal problem (1.1) is more difficult to be obtained than those in $[\mathbf{3 9}]$. In particular, we must point out that the exact boundary behavior of susceptible $S(\xi)$ at $\xi=+\infty$ is more difficult to be obtained than the local SIR model (1.3). The main reason is that we do not know whether $S(\xi)$ is monotone or non-monotone due to the effect of nonlocal dispersal compared to problem (1.3) in [35].

In summary, we shall prove that, if $R_{0}=\beta / \gamma>1$, then there exists a critical velocity $c_{*}$ such that, for each $c>c_{*}$, system (1.1) admits nontrivial traveling wave solutions with wave speed $c$ and no nontrivial traveling wave solutions of (1.1) for $0<c<c_{*}$, and, if $R_{0}=\beta / \gamma \leq 1$, no nontrivial traveling wave solutions exist for every speed $c \geq 0$. 
This paper is organized as follows. In Section 2, we prove the existence of nontrivial traveling wave solutions by Schauder's fixed theorem. The nonexistence of traveling waves is obtained in Section 3 by applying the two-sided Laplace transform, which is first introduced by Carr and Chmaj [5] and further used by Wang et al. [36-38].

2. Existence of traveling waves. In this section, we shall consider the existence of traveling wave solutions of system (1.1). Since the third equation of (1.1) is relatively independent, we only consider the first two equations. Let $\xi=x+c t$. Then the traveling wave equation of system (1.1) is as follows:

$$
\left\{\begin{array}{l}
c S^{\prime}(\xi)=d_{1}(J * S(\xi)-S(\xi))-\frac{\beta S(\xi) I(\xi)}{S(\xi)+I(\xi)}, \\
c I^{\prime}(\xi)=d_{2}(J * I(\xi)-I(\xi))+\frac{\beta S(\xi) I(\xi)}{S(\xi)+I(\xi)}-\gamma I(\xi) .
\end{array}\right.
$$

Assume that the initial disease-free equilibrium is $\left(S_{0}, 0\right)$. We intend to find solutions $(S(\xi), I(\xi))$ which are nonnegative and satisfy the following boundary conditions:

$$
S(-\infty)=S_{0}, \quad \lim _{\xi \rightarrow+\infty} S(\xi):=S_{\infty}<S_{0}, \quad I( \pm \infty)=0 .
$$

Define a function as follows:

$$
f(\lambda, c)=d_{2}\left(\int_{-\infty}^{+\infty} J(y) e^{-\lambda y} d y-1\right)-c \lambda+\beta-\gamma
$$

Then, we have the following result.

Lemma 2.1. Suppose that $R_{0}:=\beta / \gamma>1$. There exists $c_{*}>0$ and $\lambda_{*}$ such that

$$
\left.\frac{\partial f(\lambda, c)}{\partial \lambda}\right|_{\left(\lambda_{*}, c_{*}\right)}=0 \quad \text { and } \quad f\left(\lambda_{*}, c_{*}\right)=0 .
$$

Furthermore, the following alternatives hold.

(i) If $c>c_{*}$, the equation $f(\lambda, c)=0$ has two positive roots, $\lambda_{1}(c)$ and $\lambda_{2}(c)$, with $0<\lambda_{1}(c)<\lambda_{*}<\lambda_{2}(c)<\widehat{\lambda}$ for some $\widehat{\lambda} \in(0,+\infty]$, and $f(\cdot, c)<0$ in $\left(\lambda_{1}(c), \lambda_{2}(c)\right)$ and $f(\cdot, c)>0$ in $\left(0, \lambda_{1}(c)\right) \cup\left(\lambda_{2}(c), \widehat{\lambda}\right)$. 
(ii) If $c<c_{*}$, we have $f(\lambda, c)>0$ for all $\lambda \in(0, \widehat{\lambda})$.

Proof. By direct calculation, we know $f(0, c)>0$ and $f(\lambda,+\infty)=$ $-\infty$ for $\lambda>0$. In addition,

$$
\frac{\partial f(\lambda, c)}{\partial c}=-\lambda<0 \quad \text { for any } \quad \lambda>0, \quad \frac{\partial f(0, c)}{\partial \lambda}=-c<0
$$

and

$$
\frac{\partial^{2} f(\lambda, c)}{\partial \lambda^{2}}=d_{2} \int_{-\infty}^{+\infty} J(x) x^{2} e^{-\lambda x} d x>0 .
$$

Then, by simple analysis theory, we can obtain these results.

In the following, we intend to show the existence of traveling wave solutions of (2.1) when $R_{0}>1$. Denote $\lambda_{i}(c)$ by $\lambda_{i}$, and fix $c>c_{*}$. Define functions as follows:

$$
\begin{aligned}
S_{+}(\xi) & =S_{0}, & S_{-}(\xi) & =\max \left\{S_{0}\left(1-\sigma e^{\alpha \xi}\right), 0\right\}, \\
I_{+}(\xi) & =e^{\lambda_{1} \xi}, & I_{-}(\xi) & =\max \left\{e^{\lambda_{1} \xi}\left(1-M e^{\eta \xi}\right), 0\right\},
\end{aligned}
$$

where $\sigma, \alpha, M$ and $\eta$ are all positive constants. Then we have the following results.

Lemma 2.2. Suppose $\alpha<\lambda_{1}$ is sufficiently small. Then, the function $S_{-}(\xi)$ satisfies

$$
c S^{\prime}(\xi) \leq d_{1}(J * S(\xi)-S(\xi))-\beta I_{+}(\xi)
$$

for any $\xi<(1 / \alpha) \ln (1 / \sigma)$ and $\sigma>1$ large enough.

Proof. If $\xi<(1 / \alpha) \ln (1 / \sigma)$, then $S_{-}(\xi)=S_{0}\left(1-\sigma e^{\alpha \xi}\right)$. We need to verify that

$$
c S_{-}^{\prime}(\xi) \leq d_{1}\left(J * S_{-}(\xi)-S_{-}(\xi)\right)-\beta I_{+}(\xi) .
$$

That is,

$$
-c \sigma \alpha S_{0} e^{\alpha \xi}-\sigma d_{1} S_{0} e^{\alpha \xi}\left(1-\int_{-\infty}^{+\infty} J(x) e^{-\alpha x} d x\right)+\beta e^{\lambda_{1} \xi} \leq 0 .
$$


Since $\xi<(1 / \alpha) \ln (1 / \sigma)$, it is sufficient to show that

$$
-c \sigma \alpha S_{0}-\sigma d_{1} S_{0}\left(1-\int_{-\infty}^{+\infty} J(x) e^{-\alpha x} d x\right)+\beta \sigma^{\left(\alpha-\lambda_{1}\right) / \alpha} \leq 0 .
$$

Keep $\sigma=1 / \alpha$, and let $\alpha$ be small enough. The above inequality holds, which implies that (2.3) holds.

Lemma 2.3. Suppose $\eta$ is small enough such that $\eta<\min \left\{\alpha, \lambda_{2}-\right.$ $\left.\lambda_{1}\right\}$ and the constant $M$ is large enough. Then, $I_{-}(\xi)$ satisfies

$$
c I^{\prime}(\xi) \leq d_{2}(J * I(\xi)-I(\xi))+\frac{\beta S_{-}(\xi) I(\xi)}{S_{-}(\xi)+I(\xi)}-\gamma I(\xi)
$$

for any $\xi<(1 / \eta) \ln (1 / M)$.

Proof. If $\xi<(1 / \eta) \ln (1 / M)$, then $I_{-}(\xi)=e^{\lambda_{1} \xi}\left(1-M e^{\eta \xi}\right)$. When $\xi \geq(1 / \alpha) \ln (1 / \sigma), S_{-}(\xi)=0$. In this case, we only need to verify that

$$
c I_{-}^{\prime}(\xi) \leq d_{2}\left(J * I_{-}(\xi)-I_{-}(\xi)\right)-\gamma I_{-}(\xi) .
$$

That is,

$$
e^{\lambda_{1} \xi}\left(\beta-f\left(\lambda_{1}, c\right)\right) \leq M e^{\left(\eta+\lambda_{1}\right) \xi}\left(\beta-f\left(\eta+\lambda_{1}, c\right)\right),
$$

where $f(\lambda, c)$ is defined as above. Note that $f\left(\lambda_{1}, c\right)=0$ and $f(\eta+$ $\left.\lambda_{1}, c\right)<0$ because $\eta<\min \left\{\lambda_{1}, \lambda_{2}-\lambda_{1}\right\}$ as $\alpha<\lambda_{1}$. Thus, following $\xi \geq(1 / \alpha) \ln (1 / \sigma)$, it is sufficient to verify that

$$
\beta \leq M e^{\eta / \alpha \ln 1 / \sigma}\left(\beta-f\left(\eta+\lambda_{1}, c\right)\right) .
$$

Consequently, we have that inequality (2.5) holds if we take

$$
M \geq \frac{\beta \sigma^{\eta / \alpha}}{\beta-f\left(\eta+\lambda_{1}, c\right)} .
$$

When $\xi<(1 / \alpha) \ln (1 / \sigma), S_{-}(\xi)=S_{0}\left(1-\sigma e^{\alpha \xi}\right)$. Then, we need to show that

$$
c I_{-}^{\prime}(\xi) \leq d_{2}\left(J * I_{-}(\xi)-I_{-}(\xi)\right)+\frac{\beta S_{-}(\xi) I_{-}(\xi)}{S_{-}(\xi)+I_{-}(\xi)}-\gamma I_{-}(\xi) .
$$


The above inequality holds if we show

$$
\begin{aligned}
& c I_{-}^{\prime}(\xi) \\
\leq & d_{2}\left(J * I_{-}(\xi)-I_{-}(\xi)\right)+(\beta-\gamma) I_{-}(\xi)-\frac{\beta\left(S_{0}-S_{-}(\xi)+I_{+}(\xi)\right) I_{-}(\xi)}{S_{0}+I_{+}(\xi)} .
\end{aligned}
$$

Applying the definition of $f(\lambda, c)$ to obtain the above inequality, it is sufficient to verify

$$
\frac{\beta\left(S_{0}-S_{-}(\xi)+I_{+}(\xi)\right) I_{-}(\xi)}{S_{0}+I_{+}(\xi)} \leq-M f\left(\lambda_{1}+\eta, c\right) e^{\left(\lambda_{1}+\eta\right) \xi} .
$$

That is,

$$
\begin{aligned}
e^{\lambda_{1} \xi}\left(S_{0} \sigma e^{\alpha \xi}+e^{\lambda_{1} \xi}\right)(1- & \left.M e^{\eta \xi}\right) \\
& \leq-\frac{M}{\beta} f\left(\lambda_{1}+\eta, c\right) e^{\left(\lambda_{1}+\eta\right) \xi}\left(S_{0}+I_{+}(\xi)\right) .
\end{aligned}
$$

It is sufficient to verify

$$
S_{0} \sigma e^{(\alpha-\eta) \xi}+e^{\left(\lambda_{1}-\eta\right) \xi} \leq-\frac{M S_{0}}{\beta} f\left(\lambda_{1}+\eta, c\right) .
$$

Since $\xi<(1 / \eta) \ln (1 / M)$ and $f\left(\lambda_{1}+\eta, c\right)<0$, we need only to show

$$
\sigma S_{0} M^{-\alpha / \eta}+M^{-\lambda_{1} / \eta} \leq-\frac{S_{0}}{\beta} f\left(\lambda_{1}+\eta, c\right)
$$

due to $\eta<\alpha$. Note that the above inequality holds if we choose $M$ large enough. So, we end our proof.

Let $M$ and $\eta$ be defined as in Lemma 2.3. Fix $0<\delta_{0}<$ $\max \left\{\left(d_{2}+\gamma\right) / \lambda_{1}, c_{*}\right\}$ and set $\lambda_{0}=\left(d_{2}+\gamma\right) / \delta_{0}$. Denote $\xi_{0}=(1 / \eta)$ $\ln \left(\lambda_{0}+\lambda_{1}\right) /\left[M\left(\lambda_{0}+\lambda_{1}+\eta\right)\right]$. We define a new function as follows:

$$
\psi_{-}(\xi)= \begin{cases}e^{\lambda_{1} \xi}\left(1-M e^{\eta \xi}\right) & \text { if } \xi \leq \xi_{0}, \\ \frac{\eta}{\lambda_{0}+\lambda_{1}+\eta}\left(\frac{\lambda_{0}+\lambda_{1}}{M\left(\lambda_{0}+\lambda_{1}+\eta\right)}\right)^{\left(\lambda_{0}+\lambda_{1}\right) / \eta} e^{-\lambda_{0} \xi} & \text { if } \xi>\xi_{0} .\end{cases}
$$

Then, we have the following result. 
Lemma 2.4. The function $\psi_{-}(\xi)$ satisfies:

$$
c \psi_{-}^{\prime}(\xi) \leq d_{2}\left(J * \psi_{-}(\xi)-\psi_{-}(\xi)\right)+\frac{\beta S_{-}(\xi) \psi_{-}(\xi)}{S_{-}(\xi)+\psi_{-}(\xi)}-\gamma \psi_{-}(\xi) .
$$

Proof. Denote $\varepsilon=\left[\eta /\left(\lambda_{0}+\lambda_{1}+\eta\right)\right]\left[\left(\lambda_{0}+\lambda_{1}\right) / M\left(\lambda_{0}+\lambda_{1}+\eta\right)\right]^{\left(\lambda_{0}+\lambda_{1}\right) / \eta}$, and define a function as follows

$$
f(\xi)=\varepsilon+M e^{\left(\lambda_{0}+\lambda_{1}+\eta\right) \xi}-e^{\left(\lambda_{0}+\lambda_{1}\right) \xi} .
$$

We claim that $f(\xi) \geq 0$ for any $\xi \geq \xi_{0}$. Obviously, $f\left(\xi_{0}\right)=0$. Thus, we need only to verify that $f^{\prime}(\xi)>0$ for any $\xi>\xi_{0}$. Since

$$
\xi>\xi_{0}=\frac{1}{\eta} \ln \frac{\lambda_{0}+\lambda_{1}}{M\left(\lambda_{0}+\lambda_{1}+\eta\right)},
$$

we have

$$
e^{\eta \xi}>\frac{\lambda_{0}+\lambda_{1}}{M\left(\lambda_{0}+\lambda_{1}+\eta\right)}
$$

Then, we can obtain that

$$
M\left(\lambda_{0}+\lambda_{1}+\eta\right) e^{\left(\lambda_{0}+\lambda_{1}+\eta\right) \xi}>\left(\lambda_{0}+\lambda_{1}\right) e^{\left(\lambda_{0}+\lambda_{1}\right) \xi} .
$$

Consequently, we get

$$
\begin{aligned}
f^{\prime}(\xi)= & M\left(\lambda_{0}+\lambda_{1}+\eta\right) e^{\left(\lambda_{0}+\lambda_{1}+\eta\right) \xi} \\
& -\left(\lambda_{0}+\lambda_{1}\right) e^{\left(\lambda_{0}+\lambda_{1}\right) \xi}>0
\end{aligned}
$$

for any $\xi>\xi_{0}$. Thus, $f(\xi) \geq 0$ for any $\xi \geq \xi_{0}$.

Note that this claim implies that $\varepsilon e^{-\lambda_{0} \xi} \geq e^{\lambda_{1} \xi}\left(1-M e^{\eta \xi}\right)$ for any $\xi \geq \xi_{0}$.

If $\xi \leq \xi_{0}$, then $\psi_{-}(\xi)=e^{\lambda_{1} \xi}\left(1-M e^{\eta \xi}\right)$. Now, we need to verify that $\psi_{-}(\xi)$ satisfies inequality (2.6). It is noticed that

$$
\begin{aligned}
J * \psi_{-}(\xi)= & \int_{-\infty}^{\xi_{0}} J(\xi-y) e^{\lambda_{1} y}\left(1-M e^{\eta y}\right) d y \\
& +\varepsilon \int_{\xi_{0}}^{+\infty} J(\xi-y) e^{-\lambda_{0} y} d y \\
\geq & \int_{-\infty}^{+\infty} J(\xi-y) e^{\lambda_{1} y}\left(1-M e^{\eta y}\right) d y
\end{aligned}
$$


Thus, it is sufficient to verify

$$
\begin{aligned}
c \psi_{-}^{\prime}(\xi) \leq & d_{2} \int_{-\infty}^{+\infty} J(\xi-y) e^{\lambda_{1} y}\left(1-M e^{\eta y}\right) d y-d_{2} \psi_{-}(\xi) \\
& +\frac{\beta S_{-}(\xi) \psi_{-}(\xi)}{S_{-}(\xi)+\psi_{-}(\xi)}-\gamma \psi_{-}(\xi)
\end{aligned}
$$

for any $\xi \leq \xi_{0}$. It follows from the proof of Lemma 2.3 that the above inequality holds. Hence, (2.6) holds.

If $\xi>\xi_{0}$, then $\psi_{-}(\xi)=\varepsilon e^{-\lambda_{0} \xi}$. We need only prove that

$$
-c \lambda_{0} \varepsilon e^{-\lambda_{0} \xi} \leq d_{2} J * \psi_{-}(\xi)+\frac{\varepsilon \beta S_{-}(\xi) e^{-\lambda_{0} \xi}}{S_{-}(\xi)+\varepsilon e^{-\lambda_{0} \xi}}-\left(d_{2}+\gamma\right) \varepsilon e^{-\lambda_{0} \xi}
$$

It is sufficient to show

$$
-c \lambda_{0} \leq \varepsilon^{-1} d_{2} e^{\lambda_{0} \xi} J * \psi_{-}(\xi)+\frac{\beta S_{-}(\xi)}{S_{0}+I_{+}(\xi)}-\left(d_{2}+\gamma\right) .
$$

Indeed,

$$
c \lambda_{0}=\frac{c\left(d_{2}+\gamma\right)}{\delta_{0}}>\frac{c\left(d_{2}+\gamma\right)}{c_{*}}>\left(d_{2}+\gamma\right) .
$$

Thus, (2.7) holds. This completes the proof.

Now, we establish the existence of traveling wave solutions of (1.1) for $R_{0}>1(\beta>\gamma)$. Take $X>\max \left\{(1 / \alpha) \ln \sigma,(1 / \eta) \ln \left[M\left(\lambda_{1}+\eta\right) / \lambda_{1}\right]\right\}$, and let

$$
\begin{aligned}
\Gamma_{X}=\left\{(\phi(\cdot), \varphi(\cdot)) \in C\left([-X, X], \mathbf{R}^{2}\right)\right. \\
\\
\left.\qquad \begin{array}{c}
\phi(-X)=S_{-}(-X), \quad \varphi(-X)=\psi_{-}(-X), \\
S_{-}(\xi) \leq \phi(\xi) \leq S_{0}, \quad \psi_{-}(\xi) \leq \varphi(\xi) \leq I_{+}(\xi) \\
\text { for any } \xi \in[-X, X] .
\end{array}\right\} .
\end{aligned}
$$

For any $(\phi(\cdot), \varphi(\cdot)) \in \Gamma_{X}$, define

$$
\widetilde{\phi}(\xi)= \begin{cases}\phi(X), & \xi>X, \\ \phi(\xi), & |\xi| \leq X, \\ S_{-}(\xi), & \xi<-X,\end{cases}
$$




$$
\widetilde{\varphi}(\xi)= \begin{cases}\varphi(X), & \xi>X \\ \varphi(\xi), & |\xi| \leq X \\ \psi_{-}(\xi), & \xi<-X\end{cases}
$$

Then, we consider the following initial value problems

$$
\begin{aligned}
c S^{\prime}(\xi)= & d_{1} \int_{-\infty}^{+\infty} J(y) \widetilde{\phi}(\xi-y) d y \\
& -\left(d_{1}+\frac{\beta \varphi(\xi)}{\phi(\xi)+\varphi(\xi)}\right) S(\xi), \\
c I^{\prime}(\xi)= & d_{2} \int_{-\infty}^{+\infty} J(y) \widetilde{\varphi}(\xi-y) d y \\
& +\left(\frac{\beta \phi(\xi)}{\phi(\xi)+\varphi(\xi)}-d_{2}-\gamma\right) I(\xi)
\end{aligned}
$$

with

$$
S(-X)=S_{-}(-X), \quad I(-X)=\psi_{-}(-X) .
$$

By the ODE theory, we have that (2.8)-(2.10) admit a unique solution $\left(S_{X}(\xi), I_{X}(\xi)\right)$ satisfying $S_{X}(\cdot) \in C^{1}([-X, X])$ and $I_{X}(\cdot) \in$ $C^{1}([-X, X])$. Then, we define an operator $\mathcal{G}=\left(\mathcal{G}_{1}, \mathcal{G}_{2}\right): \Gamma_{X} \rightarrow$ $C([-X, X])$ by $S_{X}(\xi)=\mathcal{G}_{1}[\phi, \varphi](\xi)$ and $I_{X}(\xi)=\mathcal{G}_{2}[\phi, \varphi](\xi)$ for $\xi \in[-X, X]$.

Theorem 2.5. The operator $\mathcal{G}$ maps $\Gamma_{X}$ into $\Gamma_{X}$ and is completely continuous.

Proof. Obviously, 0 is a sub-solution of (2.8). Then, by the strong maximum principle, we know $S_{X}(\xi)>0$. According to the definition of $\widetilde{\phi}(\xi)$, we have

$$
\begin{aligned}
d_{1} \int_{-\infty}^{+\infty} J(\xi-x) \widetilde{\phi}(x) d x-\left(d_{1}+\frac{\beta \varphi(\xi)}{\phi(\xi)+\varphi(\xi)}\right) & S_{0} \\
& \leq-\frac{\beta S_{0} \psi_{-}(\xi)}{S_{0}+I_{+}(\xi)} \leq 0
\end{aligned}
$$


which implies that $S_{0}$ is a super-solution of (2.8). Taking $X_{1}=$ $1 / \alpha \ln 1 / \sigma, S_{-}(\xi)=S_{0}\left(1-\sigma e^{\alpha \xi}\right)$ holds for any $\xi \in\left[-X, X_{1}\right)$. According to Lemma 2.2, we have

$$
\begin{aligned}
c S_{-}^{\prime}(\xi)-d_{1} \int_{-\infty}^{+\infty} & J(\xi-x) \widetilde{\phi}(x) d x \\
& +\left(d_{1}+\frac{\beta \varphi(\xi)}{\phi(\xi)+\varphi(\xi)}\right) S_{-}(\xi) \\
\leq & c S_{-}^{\prime}(\xi)-d_{1}\left(J * S_{-}(\xi)-S_{-}(\xi)\right)+\beta I_{+}(\xi) \leq 0,
\end{aligned}
$$

for any $\xi \in\left[-X, X_{1}\right)$. In addition, $S_{X}(-X)=S_{-}(-X)$, by the maximum principle, we have $S_{X}(\xi) \geq S_{-}(\xi)$ for any $\xi \in\left[-X, X_{1}\right]$. Consequently, it is obtained that $S_{-}(\xi) \leq S_{X}(\xi) \leq S_{0}$ for any $\xi \in$ $[-X, X]$.

Next, we consider $I_{X}(\xi)$. It is obvious that $I_{X}(\xi)>0$ for any $\xi \in$ $[-X, X]$, by the strong maximum principle. According to Lemma 2.1, it is easy to obtain that

$$
\begin{aligned}
c I_{+}^{\prime}(\xi)-d_{2} \int_{-\infty}^{+\infty} J(\xi-x) \widetilde{\varphi}(x) d x+ & \left(d_{2}+\gamma-\frac{\beta \phi(\xi)}{\phi(\xi)+\varphi(\xi)}\right) I_{+}(\xi) \\
\geq & c I_{+}^{\prime}(\xi)-d_{2}\left(J * I_{+}(\xi)-I_{+}(\xi)\right) \\
& -(\beta-\gamma) I_{+}(\xi)=0
\end{aligned}
$$

which implies that $I_{+}(\xi)$ is a super-solution of (2.9). Recalling the definition of $\widetilde{\varphi}$ and applying Lemma 2.4 , it is not difficult to show that $\psi_{-}(\xi) \leq I_{X}(\xi)$ for any $\xi \in[-X, X]$. In summary, we have $\psi_{-}(\xi) \leq I_{X}(\xi) \leq I_{+}(\xi)$ for any $\xi \in[-X, X]$. So, we have shown that $\mathcal{G}$ maps $\Gamma_{X}$ into $\Gamma_{X}$.

Finally, we show that the operator $\mathcal{G}$ is completely continuous. It follows from ODE theory that system (2.8)-(2.10) admits a unique solution $\left(S_{X}(\cdot), I_{X}(\cdot)\right)$. Moreover, $S_{X}(\cdot) \in C^{1}([-X, X])$ and $I_{X}(\cdot) \in$ $C^{1}([-X, X])$. By direct computation, we obtain that

$$
\begin{aligned}
S_{X}(\xi)= & S_{-}(-X) \exp \left\{-\frac{1}{c} \int_{-X}^{\xi}\left(d_{1}+\frac{\beta \varphi(\tau)}{\phi(\tau)+\varphi(\tau)}\right) d \tau\right\} \\
& +\frac{d_{1}}{c} \int_{-X}^{\xi} \exp \left\{-\frac{1}{c} \int_{\eta}^{\xi}\left(d_{1}+\frac{\beta \varphi(\tau)}{\phi(\tau)+\varphi(\tau)}\right) d \tau\right\} g_{\phi}^{1}(\eta) d \eta
\end{aligned}
$$


and

$$
\begin{aligned}
I_{X}(\xi)= & I_{-}(-X) \exp \left\{-\frac{1}{c} \int_{-X}^{\xi}\left(d_{2}+\gamma-\frac{\beta \phi(\tau)}{\phi(\tau)+\varphi(\tau)}\right) d \tau\right\} \\
& +\frac{d_{2}}{c} \int_{-X}^{\xi} \exp \left\{-\frac{1}{c} \int_{\eta}^{\xi}\left(d_{2}+\gamma-\frac{\beta \phi(\tau)}{\phi(\tau)+\varphi(\tau)}\right) d \tau\right\} g_{\varphi}^{2}(\eta) d \eta,
\end{aligned}
$$

where

$$
\begin{aligned}
g_{\phi}^{1}(\eta)= & \int_{-\infty}^{-X} J(\eta-y) S_{-}(y) d y \\
& +\int_{-X}^{X} J(\eta-y) \phi(y) d y \\
& +\int_{X}^{+\infty} J(\eta-y) \phi(X) d y, \\
g_{\varphi}^{2}(\eta)= & \int_{-\infty}^{-X} J(\eta-y) \psi-(y) d y \\
& +\int_{-X}^{X} J(\eta-y) \varphi(y) d y \\
& +\int_{X}^{+\infty} J(\eta-y) \varphi(X) d y .
\end{aligned}
$$

Choose $\left(\phi_{i}, \varphi_{i}\right) \in \Gamma_{X}, i=1,2$, and suppose that $S_{X, i}(\xi)=\mathcal{G}_{1}\left[\phi_{i}, \varphi_{i}\right](\xi)$ and $I_{X, i}(\xi)=\mathcal{G}_{2}\left[\phi_{i}, \varphi_{i}\right](\xi)$. Note that

$$
\begin{aligned}
\left|g_{\phi_{1}}^{1}(\eta)-g_{\phi_{2}}^{1}(\eta)\right| \leq & \left|\int_{-X}^{X} J(\eta-y)\left[\phi_{1}(y)-\phi_{2}(y)\right] d y\right| \\
& +\left|\int_{X}^{+\infty} J(\eta-y)\left[\phi_{1}(X)-\phi_{2}(X)\right] d y\right| \\
& \leq 2 \max _{y \in[-X, X]}\left|\phi_{1}(y)-\phi_{2}(y)\right|
\end{aligned}
$$

and

$$
\begin{aligned}
\left|g_{\varphi_{1}}^{2}(\eta)-g_{\varphi_{2}}^{2}(\eta)\right| \leq & \left|\int_{-X}^{X} J(\eta-y)\left[\varphi_{1}(y)-\varphi_{2}(y)\right] d y\right| \\
& +\left|\int_{X}^{+\infty} J(\eta-y)\left[\varphi_{1}(X)-\varphi_{2}(X)\right] d y\right| \\
& \leq 2 \max _{y \in[-X, X]}\left|\varphi_{1}(y)-\varphi_{2}(y)\right| .
\end{aligned}
$$


Then, by the definition of the operator $\mathcal{G}$ and equations (2.11) and (2.12), we know that $\mathcal{G}$ is continuous. Moreover, since $S_{X}$ and $I_{X}$ are all of class $C^{1}([-X, X])$ and, according to equations (2.8) and (2.9), for any $(\phi(\cdot), \varphi(\cdot)) \in \Gamma_{X}$, we know $S_{X}^{\prime}$ and $I_{X}^{\prime}$ are all bounded for any $\xi \in[-X, X]$. Thus, it is obtained that the operator $\mathcal{G}$ is compact. Finally, we have obtained that $\mathcal{G}$ is completely continuous.

Furthermore, it is easy to verify that $\Gamma_{X}$ is closed and convex. Thus, Schauder's fixed theorem implies that there exists $\left(S_{X}(\cdot), I_{X}(\cdot)\right) \in \Gamma_{X}$ such that

$$
\left(S_{X}(\xi), I_{X}(\xi)\right)=\mathcal{G}\left[S_{X}, I_{X}\right](\xi)
$$

for any $\xi \in(-X, X)$. Define

$C^{1,1}([-X, X])=\left\{u \in C^{1}([-X, X]) \mid u\right.$ and $u^{\prime}$ are Lipschitz continuous $\}$

with the norm

$$
\|u\|_{C^{1,1}([-X, X])}=\max _{x \in[-X, X]}|u|+\max _{x \in[-X, X]}\left|u^{\prime}\right|+\sup _{\substack{x, y \in[-X, X] \\ x \neq y}} \frac{\left|u^{\prime}(x)-u^{\prime}(y)\right|}{|x-y|} .
$$

Then, we list some estimates about $S_{X}$ and $I_{X}$ as follows.

Theorem 2.6. There exists a constant $\widetilde{C}>0$ such that

$$
\begin{aligned}
\left\|S_{X}\right\|_{C^{1,1}([-X, X])} & <\widetilde{C}, \\
\left\|I_{X}\right\|_{C^{1,1}([-X, X])} & <\widetilde{C}
\end{aligned}
$$

and

$$
\int_{-X}^{X} I_{X}(\xi) d \xi<\widetilde{C}
$$

for any $X>\max \left\{(1 / \alpha) \ln \sigma,(1 / \eta) \ln \left[M\left(\lambda_{1}+\eta\right) / \lambda_{1}\right], R\right\}$, where $R$ is the radius of $\operatorname{supp} J$.

Proof. By the above discussion, we know that $\left(S_{X}, I_{X}\right)$ satisfies

$$
c S_{X}^{\prime}(\xi)=d_{1} \int_{-\infty}^{+\infty} J(\xi-y) \widetilde{S}_{X}(y) d y-d_{1} S_{X}(\xi)-\frac{\beta S_{X}(\xi) I_{X}(\xi)}{S_{X}(\xi)+I_{X}(\xi)}
$$


and

$$
\begin{aligned}
c I_{X}^{\prime}(\xi)= & d_{2} \int_{-\infty}^{+\infty} J(\xi-y) \widetilde{I}_{X}(y) d y-d_{2} I_{X}(\xi) \\
& +\frac{\beta S_{X}(\xi) I_{X}(\xi)}{S_{X}(\xi)+I_{X}(\xi)}-\gamma I_{X}(\xi)
\end{aligned}
$$

for any $\xi \in(-X, X)$, where

$$
\begin{aligned}
& \widetilde{S}_{X}(\xi)= \begin{cases}S_{X}(X), & \xi>X, \\
S_{X}(\xi), & |\xi| \leq X, \\
S_{-}(\xi), & \xi<-X,\end{cases} \\
& \widetilde{I}_{X}(\xi)= \begin{cases}I_{X}(X), & \xi>X, \\
I_{X}(\xi), & |\xi| \leq X, \\
\psi_{-}(\xi), & \xi<-X .\end{cases}
\end{aligned}
$$

First, integrating (2.13) from $-X$ to $X$, we have

$$
\begin{aligned}
\int_{-X}^{X} \frac{\beta S_{X}(\xi) I_{X}(\xi)}{S_{X}(\xi)+I_{X}(\xi)} d \xi= & d_{1} \int_{-X}^{X} \int_{-\infty}^{+\infty} J(y)\left(\widetilde{S}_{X}(\xi-y)-S_{X}(\xi)\right) d y d \xi \\
& -c\left[S_{X}(X)-S_{X}(-X)\right] .
\end{aligned}
$$

An easy calculation gives

$$
\begin{aligned}
\int_{-X}^{X} \int_{-\infty}^{+\infty} J(y) & \left(\widetilde{S}_{X}(\xi-y)-S_{X}(\xi)\right) d y d \xi \\
= & \int_{-\infty}^{+\infty} J(y) \int_{-X-y}^{X-y}\left(\widetilde{S}_{X}(z)-S_{X}(z+y)\right) d z d y \\
= & \left\{\int_{-\infty}^{0} J(y) \int_{-X-y}^{X}\left(S_{X}(z)-S_{X}(z+y)\right) d z d y\right\} \\
& +\left\{\int_{0}^{+\infty} J(y) \int_{-X}^{X-y}\left(S_{X}(z)-S_{X}(z+y)\right) d z d y\right\} \\
& +\left\{\int_{-\infty}^{0} J(y) \int_{X}^{X-y}\left(S_{X}(X)-S_{X}(z+y)\right) d z d y\right. \\
& \left.+\int_{0}^{+\infty} J(y) \int_{-X-y}^{-X}\left(S_{-}(z)-S_{X}(z+y)\right) d z d y\right\} \\
= & \Gamma_{s_{1}}+\Gamma_{s_{2}}+\Gamma_{s_{3} .}
\end{aligned}
$$


Noting that $0 \leq S_{X}(\xi) \leq S_{0}$, we have

$$
\begin{aligned}
\Gamma_{s_{1}} & =\int_{-\infty}^{0} J(y) \int_{-X-y}^{X}(-y) \int_{0}^{1} S^{\prime}{ }_{X}(z+t y) d t d z d y \\
& =\int_{-\infty}^{0} J(y)(-y) \int_{0}^{1}\left(S_{X}(X+t y)-S_{X}(-X-y+t y)\right) d t d y \\
& \leq S_{0} \int_{-\infty}^{0} J(y)(-y) d y \\
\Gamma_{s_{2}} & =\int_{0}^{+\infty} J(y) \int_{-X}^{X-y}(-y) \int_{0}^{1} S^{\prime}{ }_{X}(z+t y) d t d z d y \\
& =\int_{0}^{+\infty} J(y)(-y) \int_{0}^{1}\left(S_{X}(X-y+t y)-S_{X}(-X+t y)\right) d t d y \\
& \leq S_{0} \int_{0}^{+\infty} J(y) y d y
\end{aligned}
$$

and

$$
\begin{aligned}
\Gamma_{s_{3}} \leq & \int_{-\infty}^{0} J(y) \int_{X}^{X-y} S_{X}(X) d z d y \\
& +\int_{0}^{+\infty} J(y) \int_{-X-y}^{-X} S_{-}(z) d z d y \\
\leq & S_{0} \int_{-\infty}^{0} J(y)(-y) d y+S_{0} \int_{0}^{+\infty} J(y) y d y .
\end{aligned}
$$

Thus, according to $J(x)=J(-x)$, we can obtain that

$$
\int_{-X}^{X} \frac{\beta S_{X}(\xi) I_{X}(\xi)}{S_{X}(\xi)+I_{X}(\xi)} d \xi \leq 4 d_{1} S_{0} \int_{0}^{+\infty} J(y) y d y+c S_{0} .
$$

Below, we divide two cases to obtain that some constant $C_{1}$ exists independent of $X$ such that $\int_{-X}^{X} I_{X}(\xi) d \xi<C_{1}$.

Case I. We consider the case $I_{X}^{\prime}(X)<0$. Using the fact that $I_{X}^{\prime}(-X) \geq \psi_{-}^{\prime}(-X)>0$, there exists some $\xi_{M} \in(-X, X)$ such that $I_{X}\left(\xi_{M}\right)=\max _{\xi \in[-X, X]} I_{X}(\xi)$. Hence, it follows from (2.14) that

$$
\begin{aligned}
\gamma I_{X}\left(\xi_{M}\right)= & d_{2}\left\{\int_{-\infty}^{+\infty} J\left(\xi_{M}-y\right) \widetilde{I}_{X}(y) d y-I_{X}\left(\xi_{M}\right)\right\} \\
& +\frac{\beta S_{X}\left(\xi_{M}\right) I_{X}\left(\xi_{M}\right)}{S_{X}\left(\xi_{M}\right)+I_{X}\left(\xi_{M}\right)} .
\end{aligned}
$$


Since

$$
\begin{aligned}
\int_{-\infty}^{+\infty} J\left(\xi_{M}-y\right) \widetilde{I}_{X}(y) d y & I_{X}\left(\xi_{M}\right) \\
= & \int_{-\infty}^{-X} J\left(\xi_{M}-y\right) I_{-}(y) d y \\
& +\int_{-X}^{X} J\left(\xi_{M}-y\right) I_{X}(y) d y \\
& +\int_{X}^{+\infty} J\left(\xi_{M}-y\right) I_{X}(X) d y-I_{X}\left(\xi_{M}\right) \\
\leq & \|\|_{L^{\infty}(\mathbf{R})}^{-X} \int_{-\infty}^{-} \psi_{-}(y) d y
\end{aligned}
$$

and

$$
\frac{\beta S_{X}\left(\xi_{M}\right) I_{X}\left(\xi_{M}\right)}{S_{X}\left(\xi_{M}\right)+I_{X}\left(\xi_{M}\right)} \leq \beta S_{0}
$$

we have

$$
I_{X}\left(\xi_{M}\right) \leq \frac{d_{2}\|J\|_{L^{\infty}(\mathbf{R})}}{\gamma} \int_{-\infty}^{-X} \psi_{-}(y) d y+\frac{\beta S_{0}}{\gamma}
$$

Then, integrating (2.14) from $-X$ to $X$, we have

$$
\begin{aligned}
\gamma \int_{-X}^{X} I_{X}(\xi) d \xi= & d_{2} \int_{-X}^{X}\left\{\int_{-\infty}^{+\infty} J(y) \widetilde{I}_{X}(\xi-y) d y-I_{X}(\xi)\right\} d \xi \\
& +\int_{-X}^{X} \frac{\beta S_{X}(\xi) I_{X}(\xi)}{S_{X}(\xi)+I_{X}(\xi)} d \xi \\
& -c\left[I_{X}(X)-I_{X}(-X)\right] .
\end{aligned}
$$

It is noticed that

$$
\begin{aligned}
\int_{-X}^{X}\left\{\int_{-\infty}^{+\infty} J(y) \tilde{I}_{X}(\xi-y) d y-I_{X}(\xi)\right\} d \xi \\
=\int_{-\infty}^{+\infty} J(y) \int_{-X-y}^{X-y}\left(\tilde{I}_{X}(z)-I_{X}(z+y)\right) d z d y \\
=\left\{\int_{-\infty}^{0} J(y) \int_{-X-y}^{X}\left(I_{X}(z)-I_{X}(z+y)\right) d z d y\right\}
\end{aligned}
$$




$$
\begin{aligned}
& +\left\{\int_{0}^{+\infty} J(y) \int_{-X}^{X-y}\left(I_{X}(z)-I_{X}(z+y)\right) d z d y\right\} \\
& +\left\{\int_{-\infty}^{0} J(y) \int_{X}^{X-y}\left(I_{X}(X)-I_{X}(z+y)\right) d z d y\right. \\
& \left.+\int_{0}^{+\infty} J(y) \int_{-X-y}^{-X}\left(\psi_{-}(z)-I_{X}(z+y)\right) d z d y\right\} \\
& =\Gamma_{I_{1}}+\Gamma_{I_{2}}+\Gamma_{I_{3}} .
\end{aligned}
$$

Since $I_{X}(\xi) \leq I_{X}\left(\xi_{M}\right)$ for any $\xi \in[-X, X]$, by the same computation as above, we obtain that

$$
\Gamma_{I_{1}}+\Gamma_{I_{2}} \leq 2\left\{\frac{d_{2}\|J\|_{L^{\infty}(\mathbf{R})}}{\gamma} \int_{-\infty}^{-X} \psi_{-}(y) d y+\frac{\beta S_{0}}{\gamma}\right\} \int_{0}^{+\infty} J(y) y d y
$$

Moreover, we have

$$
\begin{aligned}
\Gamma_{I_{3}} \leq & \left(\frac{d_{2}\|J\|_{L^{\infty}(\mathbf{R})}}{\gamma} \int_{-\infty}^{0} J(y)(-y) d y+1\right) \int_{-\infty}^{-X} \psi_{-}(y) d y \\
& +\frac{\beta S_{0}}{\gamma} \int_{-\infty}^{0} J(y)(-y) d y
\end{aligned}
$$

Thus, it is obtained from (2.15) and (2.16) that

$$
\begin{aligned}
\gamma \int_{-X}^{X} I_{X}(\xi) d \xi \leq & \frac{1}{\gamma}\left(\beta S_{0}+d_{2}\|J\|_{L^{\infty}(\mathbf{R})} \int_{-\infty}^{-X} \psi_{-}(y) d y\right) \\
& \times\left(c+3 d_{2} \int_{0}^{+\infty} J(y) y d y\right) \\
& +4 d_{1} S_{0} \int_{0}^{+\infty} J(y) y d y \\
& +c S_{0}+d_{2} \int_{-\infty}^{-X} \psi_{-}(z) d z .
\end{aligned}
$$

Here, since $X<\xi_{0}$, it is easy to see that $\int_{-\infty}^{-X} \psi_{-}(y) d y<C$ for some constant $C$ independent of $X$.

Case II. We now consider the case that $I_{X}^{\prime}(X) \geq 0$. Note that if $I_{X}(\xi)$ attains its maximum at an interior point $\xi_{M} \in(-X, X)$, we have 
done as in Case I. Thus, we assume $I_{X}(X)=\max _{\xi \in[-X, X]} I_{X}(\xi)$. Since $I_{X}(\xi) \in C^{1}([-X, X])$, some point sequence $\xi_{n} \in(-X, X)$ exists such that

$$
\lim _{n \rightarrow+\infty} I_{X}\left(\xi_{n}\right)=I_{X}(X)
$$

and

$$
\begin{aligned}
c I_{X}^{\prime}\left(\xi_{n}\right)= & d_{2}\left(\int_{-\infty}^{+\infty} J\left(\xi_{n}-y\right) \widetilde{I}_{X}(y) d y-I_{X}\left(\xi_{n}\right)\right) \\
& +\frac{\beta S_{X}\left(\xi_{n}\right) I_{X}\left(\xi_{n}\right)}{S_{X}\left(\xi_{n}\right)+I_{X}\left(\xi_{n}\right)}-\gamma I_{X}\left(\xi_{n}\right)
\end{aligned}
$$

holds. Note that, for any $\varepsilon>0$, there exists some $N_{0}>0$ such that for each $n>N_{0}$ we have $\left|I_{X}(X)-I_{X}\left(\xi_{n}\right)\right|<\varepsilon$ and $I_{X}^{\prime}\left(\xi_{n}\right) \geq 0$. Thus, for every $n>N_{0}$, there is

$$
\begin{aligned}
\gamma I_{X}\left(\xi_{n}\right) & \leq d_{2}\left(\int_{-\infty}^{+\infty} J\left(\xi_{n}-y\right) \widetilde{I}_{X}(y) d y-I_{X}\left(\xi_{n}\right)\right)+\beta S_{0} \\
& \leq d_{2}\|J\|_{L^{\infty}(\mathbf{R})} \int_{-\infty}^{-X} \psi_{-}(y) d y+d_{2} \varepsilon+\beta S_{0} .
\end{aligned}
$$

Now, we can pass to the limit on the above expression to get a uniform bound of $I_{X}(X)$. Then, discussing as above, we also can obtain that some constant $C_{1}$ exists independent of $X$ such that $\int_{-X}^{X} I_{X}(\xi) d \xi<C_{1}$.

It is noticed that $S_{X}(\xi) \leq S_{0}$ for any $\xi \in[-X, X]$. By simple computation, we have

$$
\begin{aligned}
\left|S^{\prime}{ }_{X}(\xi)\right| \leq & \frac{d_{1}}{c} \int_{-\infty}^{+\infty} J(\xi-y) \widetilde{S}_{X}(y) d y \\
& +\frac{d_{1}}{c} S_{X}(\xi)+\frac{\beta}{c} \frac{S_{X}(\xi) I_{X}(\xi)}{S_{X}(\xi)+I_{X}(\xi)} \\
\leq & \frac{2 d_{1}+\beta}{c} S_{0} .
\end{aligned}
$$

According to the discussion as above, we know that there exists some constant $C_{*}$ independent of $X$ such that $I_{X}(\xi)<C_{*}$ for any $\xi \in$ $[-X, X]$. Then, it follows from (2.14) that

$$
\left|I_{X}^{\prime}(\xi)\right| \leq \frac{d_{2}\|J\|_{L^{\infty}}}{c} \int_{-\infty}^{-X} \psi_{-}(y) d y+\frac{\left(2 d_{2}+\gamma\right) C_{*}}{c}+\frac{\beta}{c} S_{0} .
$$


Consequently, it follows from the above two inequalities that some constant $C_{0}$ exists independent of $X$ such that $\left|S^{\prime}{ }_{X}(\xi)\right|<C_{0}$ and $\left|I^{\prime}{ }_{X}(\xi)\right|<C_{0}$. Thus, for any $\xi, \eta \in[-X, X]$, some constant $L_{1}>0$ exists such that

$$
\left|S_{X}(\xi)-S_{X}(\eta)\right|<L_{1}|\xi-\eta| \text { and } \quad\left|I_{X}(\xi)-I_{X}(\eta)\right|<L_{1}|\xi-\eta| .
$$

On the other hand, it follows from (2.13) that

$$
\begin{aligned}
\left|S^{\prime}{ }_{X}(\xi)-S^{\prime}{ }_{X}(\eta)\right| \leq & \frac{d_{1}}{c} \mid \int_{-\infty}^{+\infty} J(\xi-y) \widetilde{S}_{X}(y) d y \\
& -\int_{-\infty}^{+\infty} J(\eta-y) \widetilde{S}_{X}(y) d y \mid \\
& +\frac{d_{1}}{c}\left|S_{X}(\xi)-S_{X}(\eta)\right| \\
& +\frac{\beta}{c}\left|\frac{S_{X}(\xi) I_{X}(\xi)}{S_{X}(\xi)+I_{X}(\xi)}-\frac{S_{X}(\eta) I_{X}(\eta)}{S_{X}(\eta)+I_{X}(\eta)}\right| \\
= & I_{1}+I_{2}+I_{3} .
\end{aligned}
$$

It is noticed that

$$
\begin{aligned}
&\left|\int_{-X}^{X}(J(\xi-y)-J(\eta-y)) S_{X}(y) d y\right| \\
&=\left|\int_{\xi-X}^{\xi+X} J(z) S_{X}(\xi-z) d z-\int_{\eta-X}^{\eta+X} J(z) S_{X}(\eta-z) d z\right| \\
&=\mid \int_{\eta+X}^{\xi+X} J(z) S_{X}(\xi-z) d z-\int_{\eta-X}^{\xi-X} J(z) S_{X}(\eta-z) d z \\
& \\
&+\int_{\xi-X}^{\eta+X} J(z)\left(S_{X}(\xi-z)-S_{X}(\eta-z)\right) d z \mid \\
& \leq\left(2\|J\|_{L^{\infty}} S_{0}+L_{1}\right)|\xi-\eta| .
\end{aligned}
$$

According to assumption $(\mathrm{J})$, we know $J$ is Lipschitz continuous. Thus, we have

$$
I_{1}=\frac{d_{1}}{c}\left|\int_{-\infty}^{+\infty} J(\xi-y) \widetilde{S}_{X}(y) d y-\int_{-\infty}^{+\infty} J(\eta-y) \widetilde{S}_{X}(y) d y\right|
$$




$$
\begin{aligned}
= & \frac{d_{1}}{c} \mid \int_{-\infty}^{-X}(J(\xi-y)-J(\eta-y)) S_{-}(y) d y \\
& +\int_{-X}^{X}(J(\xi-y)-J(\eta-y)) S_{X}(y) d y \\
& +\int_{X}^{+\infty}(J(\xi-y)-J(\eta-y)) S_{X}(X) d y \mid \\
\leq & \frac{d_{1}}{c}\left\{S_{0}\left|\int_{\xi+X}^{\eta+X} J(z) d z\right|+\left|\int_{\eta-X}^{\xi-X} J(y) d y\right| S_{0}\right. \\
& +\sigma S_{0} \int_{-\infty}^{-X}|J(\xi-y)-J(\eta-y)| e^{\alpha y} d y \\
& \left.+\left|\int_{-X}^{X}(J(\xi-y)-J(\eta-y)) S_{X}(y) d y\right|\right\} \\
\leq & \frac{d_{1}}{c}\left(\sigma L S_{0}+4 S_{0}\|J\|_{L^{\infty}}+L_{1}\right)|\xi-\eta|
\end{aligned}
$$

for the Lipschitz constant $L$ of $J$. In addition, a simple computation yields

$$
\begin{aligned}
I_{3} & =\frac{\beta}{c}\left|\frac{S_{X}(\xi) I_{X}(\xi)}{S_{X}(\xi)+I_{X}(\xi)}-\frac{S_{X}(\eta) I_{X}(\eta)}{S_{X}(\eta)+I_{X}(\eta)}\right| \\
& \leq \frac{\beta}{c}\left[\left|I_{X}(\xi)-I_{X}(\eta)\right|+\left|S_{X}(\xi)-S_{X}(\eta)\right|\right] .
\end{aligned}
$$

Then, there exists some constant $L_{2}>0$ such that

$$
\left|S^{\prime}{ }_{X}(\xi)-S^{\prime}{ }_{X}(\eta)\right|<L_{2}|\xi-\eta|
$$

for any $\xi, \eta \in[-X, X]$. In addition, it follows from

$$
\begin{aligned}
\mid \int_{-\infty}^{+\infty} J(y) \tilde{I}_{X}(\xi-y) & d y-\int_{-\infty}^{+\infty} J(y) \tilde{I}_{X}(\eta-y) d y \mid \\
\leq & \int_{-\infty}^{-X}|J(\xi-y)-J(\eta-y)| \psi_{-}(y) d y \\
& +\int_{-X}^{X}|J(\xi-y)-J(\eta-y)| I_{X}(y) d y \\
& +C_{*}\left|\int_{\eta-X}^{\xi-X} J(z) d z\right|
\end{aligned}
$$




$$
\begin{aligned}
& \leq\left(L \int_{-\infty}^{-X} \psi_{-}(y) d y\right. \\
& \left.\quad+L \int_{-X}^{X} I_{X}(y) d y+C_{*}\|J\|_{L^{\infty}(\mathbf{R})}\right)|\xi-\eta|
\end{aligned}
$$

and the estimate of $I_{3}$ that

$$
\left|I_{X}^{\prime}(\xi)-I_{X}^{\prime}(\eta)\right|<L_{2}|\xi-\eta|
$$

for any $\xi, \eta \in[-X, X]$. So far, we have shown that some constant $\widetilde{C}>0$ exists independent of $X$ such that

$$
\left\|S_{X}\right\|_{C^{1,1}([-X, X])}<\widetilde{C}, \quad\left\|I_{X}\right\|_{C^{1,1}([-X, X])}<\widetilde{C} .
$$

Thus, we have completed the whole proof.

Now, choose a sequence $\left\{X_{n}\right\}_{1}^{\infty}$ satisfying

$$
X_{n}>\max \left\{\frac{1}{\alpha} \ln \sigma, \frac{1}{\eta} \ln \frac{M\left(\lambda_{1}+\eta\right)}{\lambda_{1}}, R\right\}
$$

for each $n$ and $\lim _{n \rightarrow+\infty} X_{n}=+\infty$. We know some $\left(S_{X_{n}}, I_{X_{n}}\right) \in \Gamma_{X_{n}}$ exists satisfying Theorem 2.6. Thus, there exists a subsequence $\left\{X_{n_{k}}\right\}$ by the diagonal extraction argument such that $X_{n_{k}} \rightarrow+\infty$ as $k \rightarrow+\infty$ and

$$
S_{X_{n_{k}}} \longrightarrow \widetilde{S} \text { and } I_{X_{n_{k}}} \longrightarrow \widetilde{I} \text { in } C_{\mathrm{loc}}^{1}(\mathbf{R}) \text { as } k \rightarrow+\infty \text {. }
$$

Since $J$ is compactly supported on $\mathbf{R}$, using the Lebesgue dominated convergence theorem, we have

$$
\begin{aligned}
\lim _{k \rightarrow+\infty} \int_{-\infty}^{+\infty} J(\xi-y) \widetilde{S}_{X_{n_{k}}}(y) d y & =\int_{-\infty}^{+\infty} J(\xi-y) \widetilde{S}(y) d y \\
& =J * \widetilde{S}(\xi),
\end{aligned}
$$

and

$$
\begin{aligned}
\lim _{k \rightarrow+\infty} \int_{-\infty}^{+\infty} J(\xi-y) \widetilde{I}_{X_{n_{k}}}(y) d y & =\int_{-\infty}^{+\infty} J(\xi-y) \widetilde{I}(y) d y \\
& =J * \widetilde{I}(\xi),
\end{aligned}
$$


for any $\xi \in \mathbf{R}$. Moreover, we have that $(\widetilde{S}, \widetilde{I})$ satisfies $(2.1)$ and

$$
\begin{aligned}
S_{-}(\xi) & <\widetilde{S}(\xi)<S_{0}, \\
\psi_{-}(\xi) & <\widetilde{I}(\xi)<I_{+}(\xi), \\
\int_{-\infty}^{+\infty} \widetilde{I}(\xi) d \xi & <+\infty .
\end{aligned}
$$

By the definition of $S_{-}(\xi)$, it is easy to obtain that $\widetilde{S}(-\infty)=S_{0}$. In addition, since $\int_{-\infty}^{+\infty} \widetilde{I}(\xi) d \xi<+\infty$ and $\widetilde{I}^{\prime}(\xi)$ is uniformly bounded according to the proof of Theorem 2.6, we have $\widetilde{I}( \pm \infty)=0$. Moreover, it is obvious that

$$
0 \leq \liminf _{\xi \rightarrow+\infty} \widetilde{S}(\xi) \leq \limsup _{\xi \rightarrow+\infty} \widetilde{S}(\xi) \leq S_{0} .
$$

Then, we have the following result.

Theorem 2.7. Suppose $R_{0}=\beta / \gamma>1$. If $c>c_{*}$, then a nonnegative traveling wave solution $(\widetilde{S}(x+c t), \widetilde{I}(x+c t))$ of $(2.1)$ exists satisfying (2.2).

Proof. By the above discussion, we need only prove that there exists some $S_{\infty}$ such that $\lim _{\xi \rightarrow+\infty} \widetilde{S}(\xi)=S_{\infty}$. Firstly, we claim that $\liminf _{\xi \rightarrow+\infty} \widetilde{S}(\xi)<S_{0}$. Otherwise, we have $\lim _{\xi \rightarrow+\infty} \widetilde{S}(\xi)=S_{0}$. Then, integrating the first equation of (2.1) from $-x$ to $x$ yields

$$
\begin{aligned}
c[\widetilde{S}(x)-\widetilde{S}(-x)] & d_{1} \int_{-\infty}^{+\infty} J(y)(-y) \int_{0}^{1}(\widetilde{S}(x-t y)-\widetilde{S}(-x-t y)) d t d y \\
& -\int_{-x}^{x} \frac{\beta \widetilde{S}(\xi) \widetilde{I}(\xi)}{\widetilde{S}(\xi)+\widetilde{I}(\xi)} d \xi .
\end{aligned}
$$

Letting $x \rightarrow+\infty$, we obtain that

$$
\int_{-\infty}^{+\infty} \frac{\beta \widetilde{S}(\xi) \widetilde{I}(\xi)}{\widetilde{S}(\xi)+\widetilde{I}(\xi)} d \xi=0,
$$

which contradicts $\int_{-\infty}^{+\infty}(\beta \widetilde{S}(\xi) \widetilde{I}(\xi)) /(\widetilde{S}(\xi)+\widetilde{I}(\xi)) d \xi>0$. 
Now, assume $\lim \sup _{\xi \rightarrow+\infty} \widetilde{S}(\xi)>\liminf \operatorname{lit}_{\xi \rightarrow+\infty} \widetilde{S}(\xi)$ to the contrary. Then, some point sequence $\xi_{n}$ exists satisfying $\xi_{n} \rightarrow+\infty$ as $n \rightarrow+\infty$ such that $\lim _{n \rightarrow+\infty} \widetilde{S}\left(\xi_{n}\right)=\lim \sup _{\xi \rightarrow+\infty} \widetilde{S}(\xi):=\sigma_{1}$ and $\widetilde{S}^{\prime}\left(\xi_{n}\right)=0$. Furthermore, $\lim _{n \rightarrow+\infty} J * \widetilde{S}\left(\xi_{n}\right) \leq \sigma_{1}$. And $\eta_{n}$ exists satisfying $\eta_{n} \rightarrow$ $+\infty$ as $n \rightarrow+\infty$ such that $\lim _{n \rightarrow+\infty} \widetilde{S}\left(\eta_{n}\right)=\liminf _{\xi \rightarrow+\infty} \widetilde{S}(\xi):=\sigma_{2}$ and $\widetilde{S}^{\prime}\left(\eta_{n}\right)=0$. Moreover, $\liminf \inf _{n \rightarrow \infty} J * S\left(\eta_{n}\right) \geq \sigma_{2}>0$. In view of

$$
0=c \widetilde{S}^{\prime}\left(\xi_{n}\right)=d_{1}\left(J * \widetilde{S}\left(\xi_{n}\right)-\widetilde{S}\left(\xi_{n}\right)\right)-\frac{\beta \widetilde{S}\left(\xi_{n}\right) \widetilde{I}\left(\xi_{n}\right)}{\widetilde{S}\left(\xi_{n}\right)+\widetilde{I}\left(\xi_{n}\right)},
$$

letting $n \rightarrow+\infty$, we can obtain that $\lim _{n \rightarrow+\infty} J * \widetilde{S}\left(\xi_{n}\right)=\sigma_{1}$.

Let $S_{n}(y)=\widetilde{S}\left(\xi_{n}+y\right)$. Next, we want to show that $S_{n}(y) \rightarrow \sigma_{1}$ for any $y \in \operatorname{supp} J=\Omega$. Take $\delta>0$ small enough, and let $\Omega_{\delta}=$ $\Omega \cap\left\{\lim _{n \rightarrow+\infty} S_{n}(y)<\sigma_{1}-\delta\right\}$. Then

$$
\begin{aligned}
\sigma_{1} & =\lim _{n \rightarrow+\infty} \int_{\Omega} J(y) S_{n}(y) d y \\
& \leq \limsup _{n \rightarrow+\infty} \int_{\Omega \backslash \Omega_{\delta}} J(y) S_{n}(y) d y+\limsup _{n \rightarrow+\infty} \int_{\Omega_{\delta}} J(y) S_{n}(y) d y \\
& \leq \sigma_{1} \int_{\Omega \backslash \Omega_{\delta}} J(y) d y+\left(\sigma_{1}-\delta\right) \int_{\Omega_{\delta}} J(y) d y \\
& =\sigma_{1}-\delta \int_{\Omega_{\delta}} J(y) d y,
\end{aligned}
$$

which implies $m\left(\Omega_{\delta}\right)=0$ for $\delta>0$ small enough. Here, $m(\cdot)$ denotes the measure. Thus, we obtain that $S_{n}(y) \rightarrow \sigma_{1}$ almost everywhere in $\Omega$. However, $\left\{S_{n}\right\}$ is an equicontinuous family, so the convergence is everywhere in $\Omega$. That is, $\lim _{n \rightarrow+\infty} S_{n}(y)=\sigma_{1}$ for any $y \in \Omega$.

Since $J \in C^{1}, R^{\prime}>0$ and $\delta^{\prime}>0$ must exist with $R^{\prime} \geq \delta^{\prime}$ such that

$$
\left[R^{\prime}-\delta^{\prime}, R^{\prime}+\delta^{\prime}\right] \cup\left[-R^{\prime}-\delta^{\prime},-R^{\prime}+\delta^{\prime}\right] \subset \Omega .
$$

Let $\xi_{n}^{ \pm}=\xi_{n} \pm R^{\prime}$. Using similar arguments, we get

$$
\widetilde{S}\left(\xi_{n}^{ \pm}+y\right) \longrightarrow \sigma_{1} \quad \text { as } n \rightarrow \infty \text { for any } y \in \Omega .
$$

In particular, $\widetilde{S}\left(\xi_{n}+y\right) \rightarrow \sigma_{1}$ as $n \rightarrow \infty$ if $y \in\left[-\delta^{\prime}, \delta^{\prime}\right]$. Repeating the above procedures, we obtain

$$
\widetilde{S}\left(\xi_{n}+y\right) \longrightarrow \sigma_{1} \quad \text { as } n \rightarrow \infty
$$


for any $y \in[-R, R]$, where $R>0$ denotes the radius of $\Omega$.

On the other hand, it follows from $\widetilde{I}(+\infty)=0$ and

$$
J * \widetilde{S}\left(\eta_{n}\right)=\widetilde{S}\left(\eta_{n}\right)+\frac{\beta \widetilde{S}\left(\eta_{n}\right) \widetilde{I}\left(\eta_{n}\right)}{\widetilde{S}\left(\eta_{n}\right)+\widetilde{I}\left(\eta_{n}\right)}
$$

that $\lim _{n \rightarrow \infty} J * \widetilde{S}\left(\eta_{n}\right)=\sigma_{2}<\sigma_{1}$. Using similar arguments, we can prove that

$$
\widetilde{S}\left(\eta_{n}+y\right) \longrightarrow \sigma_{2} \quad \text { as } n \rightarrow \infty
$$

for any $y \in \Omega$. Consequently, we have

$$
\widetilde{S}\left(\eta_{n}+y\right) \longrightarrow \sigma_{2} \text { as } n \rightarrow \infty \text { for any } y \in[-R, R] .
$$

Note that $\int_{\eta_{n}}^{\xi_{n}}[\widetilde{S}(\xi) \widetilde{I}(\xi)] /[\widetilde{S}(\xi)+\widetilde{I}(\xi)] d \xi \rightarrow 0$ as $n \rightarrow \infty$. Integrating the two sides of the first equation of (2.1) from $\eta_{n}$ to $\xi_{n}$, we have

$$
\begin{aligned}
0< & c\left(\sigma_{1}-\sigma_{2}\right) \\
= & c \lim _{n \rightarrow \infty}\left[\widetilde{S}\left(\xi_{n}\right)-\widetilde{S}\left(\eta_{n}\right)\right] \\
= & d_{1} \lim _{n \rightarrow \infty} \int_{\eta_{n}}^{\xi_{n}}[J * \widetilde{S}(\xi)-\widetilde{S}(\xi)] d \xi \\
& -\lim _{n \rightarrow \infty} \int_{\eta_{n}}^{\xi_{n}} \frac{\beta \widetilde{S}(\xi) \widetilde{I}(\xi)}{\widetilde{S}(\xi)+\widetilde{I}(\xi)} d \xi \\
= & d_{1} \lim _{n \rightarrow \infty} \int_{-\infty}^{\infty} J(y) y \int_{0}^{1}\left[\widetilde{S}\left(\eta_{n}-t y\right)-\widetilde{S}\left(\xi_{n}-t y\right)\right] d t d y \\
& -\lim _{n \rightarrow \infty} \int_{\eta_{n}}^{\xi_{n}} \frac{\beta \widetilde{S}(\xi) \widetilde{I}(\xi)}{\widetilde{S}(\xi)+\widetilde{I}(\xi)} d \xi \\
= & 0,
\end{aligned}
$$

which yields a contradiction. Hence, we have

$$
\liminf _{\xi \rightarrow \infty} \widetilde{S}(\xi)=\limsup _{\xi \rightarrow \infty} \widetilde{S}(\xi):=S_{\infty}<S_{0}
$$

This completes the proof. 
Remark 2.8. Note that the solution $S(\xi)$ of (1.3) is monotone in [35]. However, due to the appearance of the nonlocal dispersal operator in our problem (1.3), we don't know whether $S(\xi)$ is monotone or not; even the kernel function is symmetric. Also, for the case $R_{0}>1(\beta>\gamma)$ and $c=c_{*}$, the existence of traveling waves remains open. We shall leave these problems for further research.

3. Nonexistence of traveling waves. In this section, we prove that, if $R_{0}>1(\beta>\gamma)$ and $c<c_{*}$, then there does not exist a nontrivial and nonnegative traveling wave solution pair for (2.1). In addition, we will show there do not always exist traveling wave solutions as $R_{0} \leq 1$ $(\beta \leq \gamma)$.

Theorem 3.1. Suppose that $R_{0}=\beta / \gamma>1$. For any speed $0<c<c_{*}$, no nontrivial traveling wave solutions $(S(x+c t), I(x+c t))$ exist of system (2.1) satisfying (2.2).

Proof. On the contrary, assume $(S(x), I(x))$ is a pair of traveling wave solutions to (2.1) and (2.2). Since $(\beta S) /(S+I) \rightarrow \beta$ as $x \rightarrow-\infty$, some $x^{*}$ exists such that

$$
\frac{\beta S}{S+I}-\gamma \rightarrow \beta-\gamma>\delta=\frac{\beta-\gamma}{2}>0
$$

for all $x<x^{*}$. Thus, the second equation of (2.1) yields

$$
c I^{\prime}(x)>d_{2}(J * I(x)-I(x))+\frac{\beta-\gamma}{2} I(x)
$$

for all $x<x^{*}$. Since $I$ is integrable, we can define

$$
Q(x)=\int_{-\infty}^{x} I(y) d y
$$

Integrating (3.1) from $-\infty$ to $x$, we have

$$
\delta Q(x)<c I(x)-d_{2} \int_{-\infty}^{x}(J * I(y)-I(y)) d y .
$$


Noting that

$$
\int_{-\infty}^{x} J * I(y) d y=\int_{-\infty}^{+\infty} J(z) \int_{-\infty}^{x-z} I(\tau) d \tau d z=J * Q(x)
$$

we have

$$
\delta Q(x)<c I(x)-d_{2}(J * Q(x)-Q(x)) .
$$

Moreover, by simple computation, we can obtain that

$$
\begin{aligned}
\int_{-\infty}^{x}(J * Q(t)-Q(t)) d t \\
=\int_{-\infty}^{x} \int_{-\infty}^{+\infty} J(z)(Q(t-z)-Q(t)) d z d t \\
=\int_{-\infty}^{x} \int_{-\infty}^{+\infty}(-z) J(z) \int_{0}^{1} Q^{\prime}(t-\theta z) d \theta d z d t \\
=\int_{-\infty}^{+\infty} J(z)(-z) \int_{0}^{1} Q(x-\theta z) d \theta d z
\end{aligned}
$$

Then, an integration of (3.2) yields

$$
\delta \int_{-\infty}^{x} Q(y) d y<c Q(x)+d_{2} \int_{-\infty}^{+\infty} J(z) z \int_{0}^{1} Q(x-\theta z) d \theta d z
$$

It is noticed that $z Q(x-\theta z)$ is non-increasing with respect to $\theta \in[0,1]$. Thus, we have $z Q(x-\theta z) \leq z Q(x)$. Consequently, the following holds:

$$
\delta \int_{-\infty}^{x} Q(y) d y \leq c Q(x)
$$

Note that

$$
\begin{aligned}
\delta \int_{-\infty}^{x} Q(y) d y & =\delta \int_{0}^{+\infty} Q(x-y) d y \\
& \geq \delta \int_{0}^{\tau} Q(x-y) d y \\
& >\delta \tau Q(x-\tau)
\end{aligned}
$$


for $\tau>0$. Thus, there exists $\tau>0$ such that $Q(x-\tau)<1 / 2 Q(x)$. Denote $\mu_{0}=(\ln 2) / \tau>0$. Letting $L(x)=Q(x) e^{-\mu_{0} x}$, it is easy to verify that $L(x-\tau)<L(x)$ for all $x<x^{*}$, which implies that $L(x)$ is bounded as $x \rightarrow-\infty$. It is noticed that

$$
c I^{\prime}(x) \leq d_{2}(J * I(x)-I(x))+\beta I(x) .
$$

Then, we conclude that $I(x) e^{-\mu_{0} x}$ and $I^{\prime}(x) e^{-\mu_{0} x}$ are all bounded as $x \rightarrow-\infty$. Since $I(\xi)$ is bounded, we have

$$
\sup _{x \in \mathbf{R}}\left\{I(x) e^{-\mu_{0} x}\right\}<\infty, \quad \sup _{x \in \mathbf{R}}\left\{I^{\prime}(x) e^{-\mu_{0} x}\right\}<\infty .
$$

Moreover, since $I /(I+S) \leq 1$ and $S(x)+I(x) \rightarrow S_{0}$ as $x \rightarrow-\infty$, it is known that $\left(e^{-\mu_{0} x} I(x)\right) /(S(x)+I(x))$ is also uniformly bounded on the real line. Below, we introduce a two-sided Laplace transform on the second equation of (2.1):

$$
f(\mu, c) \int_{-\infty}^{+\infty} I(x) e^{-\mu x} d x=\int_{-\infty}^{+\infty} e^{-\mu x} \frac{\beta I^{2}(x)}{S(x)+I(x)} d x,
$$

where $\mu \in\left(0, \mu_{0}\right)$ and $f(\mu, c)$ is defined in Section 2. In fact, since $e^{-\mu_{0} x} I(x) /(S(x)+I(x))$ is uniformly bounded on the real line, the two-side Laplace integrals can be analytically continued to the whole right half plane. Otherwise, the integral on the right has a singularity at $\mu=\mu^{*}$, and it is analytic for all $\mu<\mu^{*}$ (see [41]). But, the integral on the right is actually analytic for all $\mu<\mu_{0}+\mu^{*}$, which is a contradiction. Moreover, by the definition of $f(\mu, c)$, we know $f(\mu, c)>0$ for all $\mu>0$; thus, $\int_{-\infty}^{+\infty} I(x) e^{-\mu x} d x$ is analytic in the right half plane. However, according to Lemma 2.1, we know $f(\mu, c) \rightarrow+\infty$ as $\mu \rightarrow \widehat{\lambda}$. This leads to a contradiction from $(3.4)$, see $[\mathbf{3 6}, \mathbf{3 8}]$ for earlier ideas in different settings. So, we conclude that no traveling wave solutions exist which satisfy the boundary conditions.

Theorem 3.2. Suppose that $R_{0}=\beta / \gamma \leq 1$. Then, for any speed $c>0$, no traveling wave solutions $(S(x+c t), I(x+c t))$ of $(2.1)$ satisfying (2.2) exist.

Proof. For the case $R_{0}<1$, since $I(x)$ satisfies

$$
c I^{\prime}(x)=d_{2}(J * I(x)-I(x))+\frac{\beta S(x) I(x)}{S(x)+I(x)}-\gamma I(x),
$$


integrating this equation from $-\infty$ to $+\infty$, by simple computation, we have

$$
\gamma \int_{-\infty}^{+\infty} I(x) d x \leq d_{2} \int_{-\infty}^{+\infty}(J * I(x)-I(x)) d x+\beta \int_{-\infty}^{+\infty} I(x) d x .
$$

That is,

$$
\int_{-\infty}^{+\infty} I(x) d x \leq \frac{d_{2}}{\gamma+d_{2}-\beta} \int_{-\infty}^{+\infty} J * I(x) d x<\int_{-\infty}^{+\infty} I(x) d x
$$

which is a contradiction.

For the case $R_{0}=1$, that is $\beta=\gamma$, we have

$$
c I^{\prime}(x)=d_{2}(J * I(x)-I(x))+\beta\left(\frac{S(x)}{S(x)+I(x)}-1\right) I(x) .
$$

Since $I( \pm \infty)=0$ and $\int_{\mathbf{R}}(J * I(x)-I(x)) d x=0$, the following can be obtained:

$$
\beta \int_{-\infty}^{+\infty}\left(\frac{S(x)}{S(x)+I(x)}-1\right) I(x) d x=0 .
$$

Moreover, since $S(x) /[S(x)+I(x)] \leq 1$ and $I(x)$ is nonnegative, we get

$$
\left(\frac{S(x)}{S(x)+I(x)}-1\right) I(x)=0
$$

according to the continuity of $S$ and $I$. It is noted that $\operatorname{Int}(\operatorname{supp} I)$ is not empty. Then, for any $x \in \operatorname{supp} I$, we have $S(x) /[S(x)+I(x)]-1=$ 0 , which implies $I(x)=0$. This is a contradiction.

Remark 3.3. Theorems 2.7, 3.1 and 3.2 combined provide a threshold condition for the existence of traveling wave solutions in terms of the basic reproduction number $R_{0}$ and the minimal wave speed $c_{*}$. We do anticipate that $c_{*}$ is the asymptotic speed of propagation for our model(2.1) following the work described in [24], but we do not verify it. We leave it for further study.

Acknowledgments. The authors would like to thank the anonymous referee, the handling editor Professor Stig-Olof Londen and Professor Guo Lin (Lanzhou University) for their careful reading and valuable comments, which improved our original manuscript. 


\section{REFERENCES}

1. S. Ai and W. Huang, Traveling waves for a reaction-diffusion system in population dynamics and epidemiology, Proc. Roy. Soc. Edinb. 135 (2005), 663-675.

2. F. Andreu-Vaillo, J.M. Mazón, J.D. Rossi and J. Toledo-Melero, Nonlocal diffusion problems, mathematical surveys and monographs, American Mathematical Society, Providence, RI, 2010.

3. P. Bates and F. Chen, Spectral analysis and multidimensional stability of traveling waves for nonlocal Allen-Cahn equation, J. Math. Anal. Appl. 273 (2002), $45-57$.

4. P. Bates, P. Fife, X. Ren and X. Wang, Traveling waves in a convolution model for phase transitions, Arch. Rat. Mech. Anal. 138 (1997), 105-136.

5. J. Carr and A. Chmaj, Uniqueness of travelling waves for nonlocal monostable equations, Proc. Amer. Math. Soc. 132 (2004), 2433-2439.

6. F. Chen, Stability and uniqueness of traveling waves for system of nonlocal evolution equations with bistable nonlinearity, Discr. Cont. Dynam. Syst. 24 (2009), $659-673$.

7. X. Chen, Existence, uniqueness and asymptotic stability of travelling waves in non-local evolution equations, Adv. Diff. Equat. 2 (1997), 125-160.

8. J. Coville, J. Dávila and S. Martínez, Nonlocal anisotropic dispersal with monostable nonlinearity, J. Diff. Equat. 244 (2008), 3080-3118.

9. - Existence and uniqueness of solutions to a nonlocal equation with monostable nonlinearity, SIAM J. Math. Anal. 39 (2008), 1693-1709.

10. J. Coville and L. Dupaigne, On a nonlocal reaction diffusion equation arising in population dynamics, Proc. Roy. Soc. Edinb. 137 (2007), 727-755.

11. P. De Mottoni, E. Orlandi and A. Tesei, Asymptotic behavior for a system describing epidemics with migration and spatial spread of infection, Nonlin. Anal. 3 (1979), 663-675.

12. A. Ducrot and P. Magal, Travelling wave solutions for an infection-age structured model with diffusion, Proc. Roy. Soc. Edinb. 139 (2009), 459-482.

13. A. Ducrot, P. Magal and S. Ruan, Travelling wave solutions in multigroup age-structure epidemic models, Arch. Rat. Mech. Anal. 195 (2010), 311-331.

14. Y. Hosono and B. Ilyas, Travelling waves for a simple diffusive epidemic model, Math. Model Meth. Appl. Sci. 5 (1994), 935-966.

15. W. Huang, Traveling waves for a biological reaction-diffusion model, J. Dynam. Diff. Eqns. 16 (2004), 745-765.

16. V. Hutson, S. Martinez, K. Mischailow and G.T. Vickers, The evolution of dispersal, J. Math. Biol. 47 (2003), 483-517.

17. C.Y. Kao, Y. Lou and W. Shen, Random dispersal vs. non-local dispersal, Discr. Cont. Dynam. Syst. 26 (2010), 551-596.

18. - Evolution of mixed dispersal in periodic environments, Discr. Cont. Dynam. Syst. 17 (2012), 2047-2072.

19. W.O. Kermack and A.G. McKendrick, A contribution to the mathematical theory of epidemics, Proc. Roy. Soc. Lond. 115 (1927), 700-721. 
20. W.-T. Li, G. Lin and S. Ruan, Existence of travelling wave solutions in delayed reaction-diffusion systems with applications to diffusion-competition systems, Nonlinerity 19 (2006), 1253-1273.

21. W.-T. Li, Y.-J. Sun and Z.-C. Wang, Entire solutions in the FisherKPP equation with nonlocal dispersal, Nonlin. Anal. Real Word Appl. 11 (2010), 2302-2313.

22. G. Lin, W.-T. Li and M.-J. Ma, Traveling wave solutions in delayed reaction diffusion systems with applications to multi-species models, Discr. Cont. Dynam. Syst. 13 (2010), 393-414.

23. S. Ma, Travelling wavefronts for delayed reaction-diffusion systems via a fixed point theorem, J. Diff. Eqns. 171 (2001), 294-314.

24. J.D. Murray, Mathematical biology, II, Spatial models and biomedical applications, Third edition, Interdisc. Appl. Math. 18, Springer-Verlag, New York, 2003.

25. S. Pan, Traveling wave fronts of delayed non-local diffusion systems without quasimonotonicity, J. Math. Anal. Appl. 346 (2008), 415-424.

26. - Traveling wave fronts in an epidemic model with nonlocal diffusion and time delay, Int. J. Math. Anal. 2 (2008), 1083-1088.

27. S. Pan, W.-T. Li and G. Lin, Travelling wave fronts in nonlocal reactiondiffusion systems and applications, Z. Angew. Math. Phys. 60 (2009), 377-392.

28. - Existence and stability of traveling wavefronts in a nonlocal diffusion equation with delay, Nonlin. Anal. 72 (2010), 3150-3158.

29. S. Ruan and D. Xiao, Stability of steady states and existence of travelling waves in a vector-disease model, Proc. Roy. Soc. Edinb. 134 (2004), 991-1011.

30. W. Shen and A. Zhang, Spreading speeds for monostable equations with nonlocal dispersal in space periodic habitats, J. Differ. Eqns. 15 (2010), 747-795.

31. Y.-J. Sun, W.-T. Li and Z.-C. Wang, Entire solutions in nonlocal dispersal equations with bistable nonlinearity, J. Differ. Eqns. 251 (2011), 551-581.

32. - Traveling waves for a nonlocal anisotropic dispersal equation with monostable nonlinearity, Nonlin. Anal. 74 (2011), 814-826.

33. J.-W. Sun, W.-T. Li and F.-Y. Yang, Approximate the Fokker-Planck equation by a class of nonlocal dispersal problems, Nonlin. Anal. 74 (2011), 3501-3509.

34. X. Wang, Metastability and stability of patterns in a convolution model for phase transitions, J. Differ. Eqns. 183 (2002), 434-461.

35. X.-S. Wang, H. Wang and J. Wu, Traveling waves of diffusive predatorprey systems: Disease outbreak propagation, Discr. Cont. Dynam. Syst. 32 (2012), 3303-3324.

36. Z.-C. Wang, W.-T. Li and S. Ruan, Traveling fronts in monostable equations with nonlocal delayed effects, J. Dynam. Diff. Eqns. 20 (2008), 573-607.

37. - Entire solutions in bistable reaction-diffusion equations with nonlocal delayed nonlinearity, Trans. Amer. Math. Soc. 361 (2009), 2047-2084.

38. Z.-C. Wang and J. Wu, Travelling waves of a diffusive Kermack-McKendrick epidemic model with non-local delayed transmission, Proc. Roy. Soc. Lond. 466 (2010), 237-261. 
39. Z.-C. Wang and J. Wu, Traveling waves in a bio-reactor model with stagestructure, J. Math. Anal. Appl. 385 (2012), 683-692.

40. P. Weng and X.-Q. Zhao, Spreading speed and traveling waves for a multi-type SIS epidemic model, J. Differ. Eqns. 229 (2006), 270-296.

41. D.V. Widder, The Laplace transform, Princeton University Press, Princeton, NJ, 1941.

42. D. Xiao and S. Ruan, Global analysis of an epidemic model with nonmonotone incidence rate, Math. Biosci. 208 (2007), 419-429.

43. H. Yagisita, Existence and nonexistence of traveling waves for a nonlocal monostable equation, Publ. Res. Inst. Math. Sci. 45 (2009), 925-953.

44. - Existence of traveling wave solutions for a nonlocal bistable equation: An abstract approach, Publ. Res. Inst. Math. Sci. 45 (2009), 955-979.

45. G.-B. Zhang, Traveling waves in a nonlocal dispersal population model with age-structure, Nonlin. Anal. 74 (2011), 5030-5047.

46. - Global stability of wavefronts with minimal speeds for nonlocal dispersal equations with degenerate nonlinearity, Nonlin. Anal. 74 (2011), 6518-6529.

47. G.-B. Zhang, W.-T. Li and G. Lin, Traveling waves in delayed predator-prey systems with nonlocal diffusion and stage structure, Math. Comp. Model. 49 (2009), 1021-1029.

48. G.-B. Zhang, W.-T. Li and Z.-C. Wang, Spreading speeds and traveling waves for nonlocal dispersal equations with degenerate monostable nonlinearity, J. Differ. Eqns. 252 (2012), 5096-5124.

49. L. Zhang, Existence, uniqueness and exponential stability of traveling wave solutions of some integral differential equations arising from neural networks, J. Differ. Eqns. 197 (2004), 162-196.

School of Mathematics and Statistics, Lanzhou University, Lanzhou, Gansu, 730000, P.R. China

Email address: wtli@lzu.edu.cn

School of Mathematics and Statistics, Lanzhou University, Lanzhou, Gansu, 730000, P.R. China

Email address: yangfy@lzu.edu.cn 